\title{
Time relaxation of magnetization in type-II superconductors
}

\author{
G RAVIKUMAR, P CHADDAH and K V BHAGWAT \\ Nuclear Physics Division, Bhabha Atomic Research Centre, Bombay 400085, India

\begin{abstract}
We assume that currents induced by isothermal changes of magnetic field decay logarithmically with time. Incorporating this time dependence into the critical state model, we obtain logarithmic relaxation rate of magnetization as a function of field for the case of an infinite cylinder. We compare these calculations with our earlier calculations on infinite slab geometry.
\end{abstract}

Keywords. Time relaxation; magnetization; type-II superconductors.

\section{Introduction}

An interesting feature reported for various high $T_{\mathrm{c}}$ superconductors is the rapid time decay of magnetization for the following cases (i) a ZFC sample subjected to a field $H$ which is then held constant; and (ii) a ZFC sample subjected to a field $H$ which is then switched off. In both cases the magnetization decays logarithmically with time. We have earlier calculated logarithmic relaxation rate in the above two cases in the slab geometry (Chaddah and Ravikumar 1989), following the assumption by Yeshurun et al (1988) that the phenomenon of flux creep results in shielding current density decaying as $J(t)=J(1)[1-b \ln t]$. Here we calculate the decay rate in the two cases for the case of an infinite cylinder (of radius $R$ ) where the field is along the long axis, and discuss the important geometrical effects on the decay rate. In these calculations we assume that $J_{c}(H)=J_{c}(0) \exp \left(-H / H_{0}\right)$.

\section{Logarithmic decay of magnetization}

According to Anderson's theory flux creep occurs because of thermal activation of flux lines across the pinning barrier and the flux creeps in the direction of decreasing $|H|$ (Beasley et al 1969). This causes a difference between the cases (i) and (ii) since the flux profile may not vary monotonically, between $O$ and $R$, for the field-off case. We now evaluate the decay in magnetization as the change in the field profile because of the decay in the shielding current.

\subsection{Virgin sample in constant field}

In this case the field decreases continuously from the surface to the centre of the sample and the flux always flows into the sample. Thus the magnetization decreases in magnitude. Following their earlier procedure (Chaddah and Ravikumar 1989), we 
obtain for $H<H_{1}$

$$
\begin{aligned}
\frac{\mathrm{d} M}{\mathrm{~d} \ln t}= & -\frac{2 b H_{0}^{2}}{H^{*}}\left[e^{x}(1-x)-1\right] \\
& -\frac{4 b H_{0}^{3}}{H^{* 2}}\left[p e^{x}(1-x)-e^{2 x}(1 / 4-x / 2)-(p-1 / 4)\right]
\end{aligned}
$$

and for $H>H_{1}$

$$
\begin{aligned}
\frac{\mathrm{d} M}{\mathrm{~d} \ln t}= & -\frac{2 b H_{0}^{2}}{H^{*}}\left[e^{x}(1-x)+p \ln p-p\right] \\
& -\frac{4 b H_{0}^{3}}{H^{* 2}}\left[e^{x}\left(p-e^{x} / 4\right)-x e^{x}\left(e^{x} / 2-c\right)\right. \\
& \left.-\left(e^{x}-c\right)^{2}\left[3 / 2-\ln \left(e^{x}-c\right)\right] / 2\right],
\end{aligned}
$$

where $c=H^{*} / H_{0}, \quad x=H / H_{0}, \quad p=\exp (x)-c \quad$ and $\quad H^{*}=\mu_{0} J_{c}(0) R, \quad H_{1}=H_{0} \ln$ $\left(1+H^{*} / H_{0}\right)$ is the smallest external field at which the flux fully penetrates the virgin sample.

\subsection{Decay after removal of field}

For $H<H_{\mathrm{II}}=H_{0} \ln \left(1+2 H^{*} / H_{0}\right)$, the maximum in the flux density occurs at $r=r_{0}>0$. The flux within $r<r_{0}$ flows inwards and does not contribute to the relaxation of magnetization whereas the flux which is outside the radius $r_{0}$ flows outwards and results in a decrease of magnetization. Here $r_{0}$ is given by

$$
r_{0}=R[1+2 c-\exp (x)] / 2 c .
$$

For $H<H_{11}$ we get

$$
\frac{\mathrm{d} M}{\mathrm{~d} \ln t}=-\frac{b H_{0}^{2}}{H^{*}}\left[\frac{\left(e^{x}-1\right)\left(5+4 c-e^{x}\right)}{4 c}+\frac{2(1+c)}{c} \ln \frac{2}{e^{x}+1}\right]
$$

and for $H>H_{\mathrm{II}}$

$$
-\frac{b H_{0}}{H^{*}}[2+c-2(1+1 / c) \ln (1+c)]
$$

The results of equations (1) and (2) are plotted in figure 1 for typical values $H^{*}=0.63 T$ and $H_{0}=1 T$. In the limit of $H_{0} \rightarrow \infty$, we obtain the Bean limit $J_{c}(H)=J_{c}(0)$. The ratio of the logarithmic decay rate in field-on case to that of the field-off case, in the two geometries of a slab and a cylinder, are plotted in figure 2 for the Bean limit as a function of $H$.

\section{Discussion}

As is obvious from figure 2, the geometry of the sample causes a qualitative change for $H<H_{\mathrm{Il}}$. A detailed comparison of our results in $\$ 2.1$ with those obtained earlier 


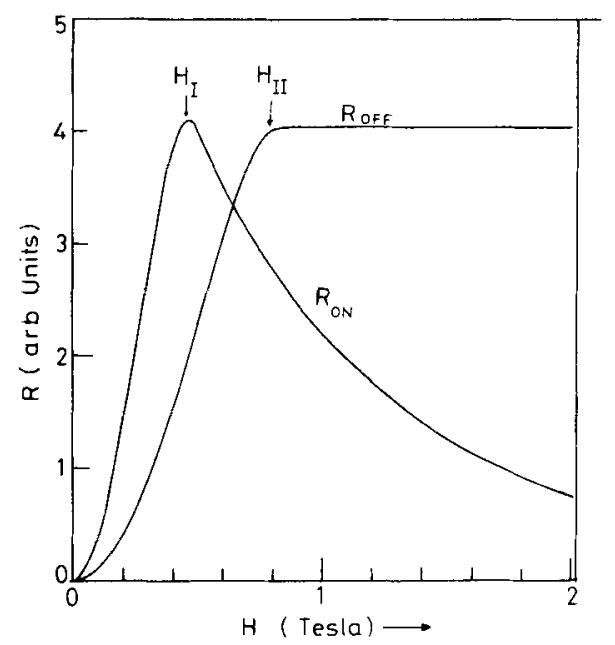

Figure 1. $|\mathrm{d} M / \mathrm{d} \ln t|$ is plotted for $H_{0}=1$ Tesla as a function of $H$. The value of $H^{*}$ is taken to be 0.63 Tesla.

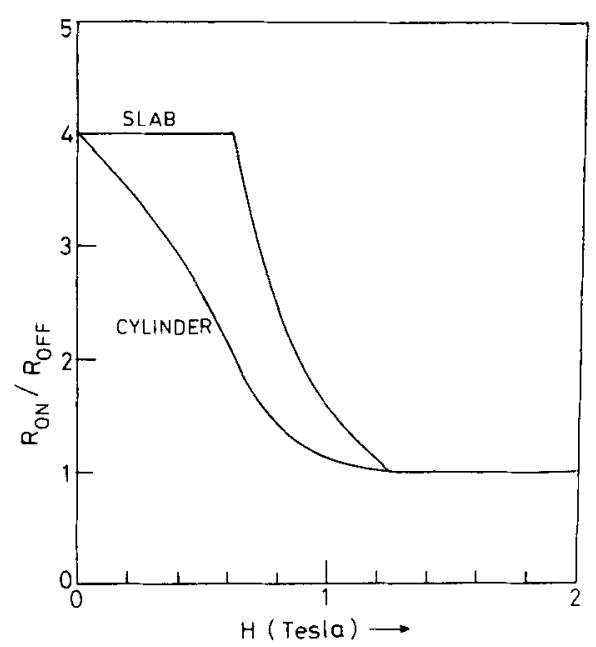

Figure 2. The ratio of $|\mathrm{d} M / \mathrm{d} \ln t|$ in field-on case to that of field-off case in slab and cylinder as a function of $H$.

(Chaddah and Ravikumar 1989) also shows that the decay rate for $H<H_{1}$ varies faster with $H$ in the cylindrical geometry. Thus both geometrical effects, and the field dependence of $J_{c}$, cause the decay rate to vary faster than $H^{2}$.

Yeshurun and Malozemoff (1988) observed that the field-cooled magnetization also decays logarithmically with time. Ravikumar (1990) extended the ideas of thermal excitation of flux lines to the case of a superconductor-cooled in external field below $T_{c}$ and has shown that the field-cooled magnetization also relaxes logarithmically with time. This will be discussed in detail in a separate paper. 


\section{References}

Beasley M R et al 1969 Phys. Rev. 181682

Chaddah P and Ravikumar G 1989 Phase Trans. 1937

Ravikumar G 1990 (to be published)

Yeshurun Y and Malozemoff A P 1988 Phys. Rev. Lett. 602202

Yeshurun Y et al 1988 Phys. Rev. B38 11828 